\title{
REFLEXÕES SOBRE A CONTRIBUIÇÃO DA CARNOT À PRIMEIRA LEI DA TERMODINÂMICA
}

Cássius K. Nascimento, João P. Braga* e José D. Fabris

Departamento de Química, Instituto de Ciências Exatas, Universidade Federal de Minas Gerais, CP 702, 31270-901 Belo Horizonte - MG

Recebido em 23/6/03; aceito em 21/11/03

\begin{abstract}
REFLECTIONS ON THE CONTRIBUTION OF CARNOT TO THE FIRST LAW OF THERMODYNAMICS. The classical interpretations of Nicolas Léonard Sadi Carnot on some physical principles involved in the operation of heat engines were fundamental to the development and formulation of the Second Law of Thermodynamics. Moreover, an accurate historical survey clearly reveals that Carnot was, by that time, also well aware about some new concepts, which were further worked out by other scientists to lead to what was, some time later, known as the mechanical equivalent of heat and the conservation of energy. Benoit Paul Émile Clapeyron recognized these original concepts in the first of Carnot's monographs, published in 1824, but no explicit citation is found in any post-Carnot classical texts dealing with the First Law of Thermodynamics, including those by Julius Robert Mayer, James Prescott Joule and Hermann Ludwig Ferdinand von Helmholtz. The main objective of the present work is to point out some historical evidences of the pioneering contribution of Carnot to the modern concept of the First Law of Thermodynamics.
\end{abstract}

Keywords: thermodynamics; mechanical equivalent of heat; energy.

\section{INTRODUÇÃO}

Nicolas Léonard Sadi Carnot (1796-1832) é um nome importante na história da Termodinâmica, por ter introduzido a idéia de transformações cíclicas e por ter provado que a mais eficiente das máquinas termodinâmicas é aquela em que todas as operações são reversíveis ${ }^{1}$. As interpretações da obra de Carnot foram de fundamental importância na formulação das leis fundamentais da Termodinâmica. Uma dessas interpretações, mais comumente expressa na idéia de que "o calor não pode passar espontaneamente de um corpo frio para um corpo mais quente", é o argumento básico da Segunda Lei da Termodinâmica, como reconhecido por Rudolph Julius Emmanuel Clausius² (1822-1888). Uma interpretação alternativa dos fundamentos da Segunda Lei foi quase simultaneamente dada por Lord Kelvin (William Thomson; 1824-1907), em 1850: "não existe máquina térmica cujo único efeito seja retirar calor de uma fonte quente e transformá-lo em trabalho" ou, ainda de maneira equivalente, "não é possível construir uma máquina térmica com rendimento de $100 \% "$.

A prospecção histórica, no entanto, remete a uma questão central: se a Segunda Lei da Termodinâmica, sob argumentos das máquinas térmicas, não poderia ser formulada sem os fundamentos da conservação da energia, Carnot deve, então, ter implícita e adequadamente equacionado a Primeira $\mathrm{Lei}^{3}$.

Uma análise crítica da descoberta simultânea da Primeira Lei da Termodinâmica é descrita no artigo de 1959, escrito por Thomas Samuel Kuhn ${ }^{4}$ (1922-1996) com o título: "Energy Conservation as an Example of Simultaneous Discovery”. No livro de Kuhn há uma atenção muito grande com os manuscritos póstumos de Carnot, escritos entre 1824 e 1832, como no apêndice da referência ${ }^{2}$. Entretanto, deve-se notar que o trabalho de Carnot de 1824 não é apreciado mais detalhadamente por Kuhn.

O principal objetivo deste trabalho é demonstrar e justificar a contribuição pioneira de Carnot para a fundamentação da Primeira Lei da

*e-mail: jpbraga@ufmg.br
Termodinâmica usando, como referência principal, a sua obra de 1824, "Réflexions Sur la Puissance Motrice du Feu et Sur les Machines Propres a Développer Cette Puissance". Buscam-se, aqui, essencialmente: (i) resposta para a questão central do real pioneirismo histórico da fundamentação científica da Primeira Lei da Termodinâmica e (ii) uma melhor análise da real contribuição de Carnot a essa lei.

\section{APRESENTAÇÃO TRADICIONAL DA PRIMEIRA LEI DA TERMODINÂMICA}

A conservação da energia na mecânica clássica é evidente no trabalho de Gottfried Wilhelm von Leibniz ${ }^{5}$ (1646-1716), ao escrever sobre o teorema do virial, em 1695. Para uma partícula de massa $m$ tem-se a energia total,

$E=p^{2} / 2 m+E_{p}(r)$,

em que as quantidades têm significados usuais. A evolução temporal da energia, para a partícula isolada, é zero $(\mathrm{d} E / \mathrm{d} t=0)$. Para um sistema de partículas, a formulação é análoga ${ }^{5}$. Cronologicamente, a Segunda Lei da Termodinâmica foi estruturada antes da Primeira Lei da Termodinâmica ser formulada, apesar da conservação da energia ser um ponto que foi observado para que se pudesse fazer uma análise conclusiva da Segunda Lei. Apesar da conservação da energia na dinâmica estar bem estabelecida desde o final do século XVII, a mesma lei na Termodinâmica teve que esperar por mais de 150 anos, para uma formulação definitiva.

Alguns textos didáticos ou científicos concedem os méritos da fundamentação da Primeira Lei a Julius Robert Mayer ${ }^{6}$ (1814-1878), a James Prescott Joule ${ }^{6,7}(1818-1889)$ e a Hermann Ludwig Ferdinand von Helmholtz ${ }^{1}$ (1821-1894). Entretanto, a análise de textos históricos (ignorando, aqui, intencionalmente Carnot, nesta etapa da presente análise), constata-se que Mayer pode ser considerado o pai da Primeira Lei da Termodinâmica. Isso pode ser sustentado pelo registro de seus estudos sobre conservação da energia, em que dá prioridade à enunciação da equivalência entre calor e "energia" mecânica. O traba- 
lho de Mayer foi publicado no Annalen der Chemie und Pharmacie, vol. 42, pag. 233, $1842^{1,8}$ e, depois, na Philosophical Magazine, com o título "Remarks on the Forces of Inorganic Nature" (série 4, vol. 24, p. 371, 1862). Joule, por suas experiências, é considerado aquele que deu fundamentação mais rigorosa à Primeira Lei da Termodinâmica. O seu estudo sobre o equivalente mecânico do calor, com descrições detalhadas dos experimentos, foi publicado no Philosophical Magazine, vol. 3, série, p. 263, 347 e 435, $1843^{1,8}$.

\section{A OBRA DE CARNOT}

Carnot escreveu seu livro durante a Primeira Revolução Industrial, época em que era necessário acelerar os processos industriais e diminuir o tempo de transporte entre a produção dos bens de consumo e os mercados consumidores, em razão do grande aumento da atividade industrial e da migração camponesa para a cidade, principalmente na Inglaterra. Trabalhos de desenvolvimento de máquinas, que começavam a ser muito utilizadas à época, como a máquina a vapor, eram de fundamental importância, mas ainda faltava muita fundamentação científica. Essa carência de trabalhos cientificamente bem fundamentados é alterada com a publicação, em 1824, do livro de Carnot, "Réflexions sur la puissance motrice du feu" (Figura 1), de fundamental importância na história da Termodinâmica. Verifica-se que Carnot estava consciente da conservação da energia e do equivalente mecânico do calor. Nas páginas 19 e 20 da edição original de seu livro ${ }^{9}$, Carnot escreve:

\begin{abstract}
"A partir de nossas primeiras operações, houve ao mesmo tempo produção de potência motriz e transferência do calórico do corpo A para o corpo B; por operações inversas, há ao mesmo tempo gasto de potência motriz e retorno do calórico do corpo $\mathrm{B}$ ao corpo A. Mas se tivéssemos agido, em cada caso, com a mesma quantidade de vapor, e se não fosse produzida nenhuma perda de potência motriz nem de calórico, a quantidade de potência motriz produzida no primeiro caso seria igual àquela gasta no segundo, e a quantidade de calórico passada, no primeiro caso, do corpo $\mathrm{A}$ ao corpo $\mathrm{B}$, seria igual à quantidade que passa de volta, no segundo, do corpo B ao corpo $\mathrm{A}$, de modo que poderíamos fazer um número indefinido de operações alternativas desse tipo sem que se tenha, no balanço global, nem potência motriz produzida nem calórico transferido de um corpo ao outro".
\end{abstract}

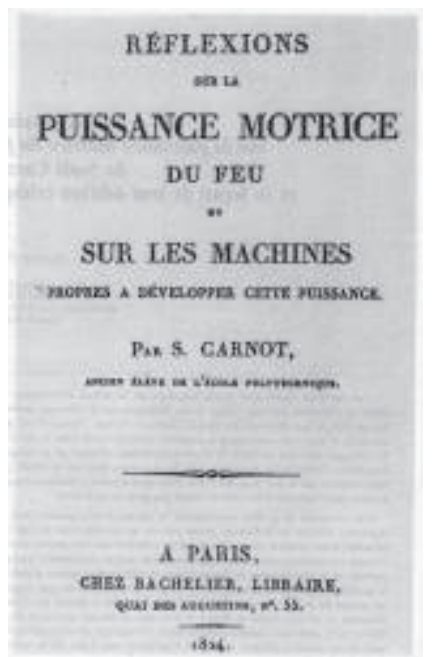

Figura 1. Fac simile da capa do livro "Réflexions sur la puissance motrice du feu - sur les machines propres a développer cette puissance" (Reflexões sobre a potência motriz do fogo) publicado em 1824
Carnot deixa claro que o calor originário da fonte de maior temperatura é transformado em trabalho e o restante do calor é absorvido pela fonte de menor temperatura. Se for realizado o processo no sentido inverso, usando-se as mesmas quantidades, serão restabelecidas as condições iniciais do sistema.

Nas páginas 38 e 39 a Primeira Lei é colocada de forma explícita9:

"Devemos ter

$$
s=e+U^{\prime \prime}
$$

A grandeza $s$ é a quantidade de calor necessária para uma expansão, $e$, a potência motriz produzida. Novamente, essa é uma formulação precisa da Primeira Lei. Carnot ainda calcula, $e=T \log V$, para o trabalho no processo isotérmico, reescrevendo a equação acima na forma $s=T \log V+U$.

A interpretação de Benoit Paul Émile Clapeyron (1799-1864), de 1834, do trabalho de $\operatorname{Carnot}^{2}$, deixa ainda mais claro esse ponto de vista. Na página 80 do "Memoir on the motive power of heat", Clapeyron escreve:

\begin{abstract}
"Das passagens anteriores, segue que a quantidade de ação mecânica e a quantidade de calor que passa de um corpo quente para um corpo frio são quantidades da mesma natureza e é possível trocar uma pela outra; do mesmo modo, na mecânica, um corpo que é capaz de cair de uma certa altura e uma massa movendo-se com uma certa velocidade são quantidades da mesma natureza, que podem ser transformadas uma na outra por meios físicos".
\end{abstract}

Essa passagem de Clapeyron deixa, de forma clara e rigorosa, a idéia central que fundamenta a Primeira Lei da Termodinâmica, como foi, antes, enunciada por Carnot.

\section{DISCUSSÃO}

Fica evidente que Carnot conhecia as bases físicas da conservação da energia. Na verdade, seria muito difícil admitir que, se desconhecesse o princípio da conservação da energia, e se não se valesse do equivalente mecânico do calor, pudesse chegar às suas conclusões sobre o funcionamento das máquinas térmicas, como o fez. Resta refletir: por que Carnot não é considerado o principal fundador da Primeira Lei da Termodinâmica? É possível que a falta do merecido reconhecimento do papel historicamente pioneiro de Carnot, na formulação das razões físicas da Primeira Lei da Termodinâmica, seja devida à precária, superficial ou inexistente interpretação de seus escritos, em época bem anterior à de Joule, Mayer e Helmholtz, quando as idéias de equivalente mecânico do calor, e mesmo do conceito mais geral de conservação da energia, já eram mais correntes e aceitos na comunidade científica. Joule e Mayer, em seus escritos sobre equivalente mecânico do calor, considerados uns dos pilares da Primeira Lei, não mencionam Carnot, apesar de suas conclusões terem sido publicadas algo como dezoito anos antes de Mayer e dezenove anos antes de Joule. Este ponto de vista é também sustentado da leitura dos trabalhos de Clapeyron, "Memoir on the motive power of heat", em 1834, e de Clausius, "On the motive power of heat, and on the laws which can be deduced from it for the theory of heat" ${ }^{\text {", em }}$ 1850 .

Os manuscritos póstumos de $\mathrm{Carnot}^{2}$, apresentados por seu irmão Lazare Hippolyte Carnot (1801-1888) na Academia de Ciências, em 1878, também confirmam a visão de Carnot sobre o Primeira Lei da Termodinâmica ${ }^{10,11}$ : "O calor não é outra coisa que a potência motriz, ou o movimento que muda de forma". 


\section{CONCLUSÕES}

De registros históricos,

(i) há claras evidências de que Carnot não somente estava consciente dos fundamentos principais da Primeira Lei da Termodinâmica, sobretudo se vistos do ponto de vista do equivalente mecânico do calor, em transformações cíclicas de máquinas térmicas, como, também formulou essa lei de forma precisa. A equação, $s=e+U$, estabelecida em 1824 não deixa dúvida desse ponto e

(ii) apesar de ter expressado com clareza suas idéias à comunidade científica da época, Carnot não é considerado, na maioria dos textos didático-científicos atuais, o primeiro a formular, claramente, a Primeira Lei da Termodinâmica. As principais razões dessa omissão histórica podem ser conseqüência da interpretação precária, superficial ou inexistente dos escritos de Carnot.

\section{AGRADECIMENTOS}

Ao CNPq, o apoio financeiro. C. K. Nascimento, especialmente, agradece à EPCAR, Barbacena, Minas Gerais, o suporte institucional.

\section{REFERÊNCIAS}

1. Magie, W. F.; A Source Book in Physics, Oxford University Press: London, 1963.

2. Mendoza, E., ed.; Reflections on the Motive Power of Fire by Sadi Carnot, and Other Papers on the Second Law of Thermodynamics by E. Clapeyron and R. Clausius, Dover Publications: New York, 1960.

3. Nascimento, C. K.; Braga, J. P.; Fabris J. D.; Resumos da $26^{a}$ Reunião Anual da Sociedade Brasileira de Química, Poços de Caldas, Brasil, 2003.

4. Kuhn, T. S.; The Essential Tension, The University of Chicago Press: Chicago, 1977.

5. Braga, J. P.; Físico-química: Aspectos Moleculares e Fenomenológicos, Ed. UFV: Viçosa, 2002.

6. Glasstone, S.; Lewis, D.; Elements of Physical Chemistry, van Nostrand Company: Princeton, 1960.

7. Barrow, G. M.; Química Física, Editorial Reverté: Barcelona, 1968.

8. Lindsay, R. B.; Energy: Historical Development of the Concept, Dowden, Hutchinson \& Ros, Inc: Stroudsburg, 1975.

9. Carnot, N. L. S.; Réflexions Sur la Puissance Motrice du Feu et Sur les Machines Propres a Développer Cette Puissance, Chez Bachelier: Paris, 1824.

10. Raman, V. V.; J. Chem. Educ.1970, 47, 331.

11. Truesdell, C. A.; The Tragicomical History of Thermodynamics, 1822-1854, Springer-Verlag: New York, 1980. 